\title{
Animal Model of Disease
}

National Cancer Institute

\section{Source}

National Cancer Institute. Animal Model of Disease. NCI Thesaurus. Code C16504.

Animal disease whose pathologic mechanisms are sufficiently similar to those of a human disease for the animal disease to serve as a model. 